\title{
SDRsim: A PC-based Simulator of Software Defined Radio
}

\author{
Yuan-Deng Chuang', Yue-Shan Chang ${ }^{2}$, Ruey-Hsiang Wu ${ }^{3}$, Shu-Ching Lin ${ }^{3}$, \\ Shyan-Ming Yuan ${ }^{3}$ \\ ${ }^{1}$ Department of Computer Science and Information Engineering \\ National Taiwan University \\ ${ }^{2}$ Department of Electronic Engineering, Minghsin Institute of Technology \\ ${ }^{3}$ Department of Computer and Information Science, National Chiao Tung University \\ d91009@csie.ntu.edu.tw, ysc@mhit.edu.tw, \\ \{gis89898, gis90573, smyuan\}@cis.nctu.edu.tw
}

\begin{abstract}
This paper represents a pc-based tool for simulation and rapid control prototyping of Software Defined Radio (SDR) technology. The tool describes a software platform implemented to facilitate the SDR software architecture at the analysis, design and implementation stages. SDRsim is a prototyping platform which provides modularity, and requires minimal implementation effort to accommodate the SDR device. It is able to demonstrate the practical use of software prototyping in reducing the complexity of product analysis and design. This simulator provides the flexibility to modify and analyze the impact of various architecture parameters and components as well as enable more detailed statistics collection than real SDR device.
\end{abstract}

\section{Introduction}

The concept of reconfigurable "software radio" [1][2] has been an active research topic which is driven initially by Military requirements for flexibility. It is best defined as the software implementation of functions inside a radio transceiver more usually implemented with analogue or digital electronic circuits, where software can be defined as a set of instructions running on a programmable processor.

Software Defined Radio (SDR) [3][4] is introduced as a new radio concept. Its objective is to use the software technology to control digital components so that a user's hand-held device may roam over different wireless networks from a building to the global and use the technologies in the fields which range from micro-cell to satellite.

SDR integrates the digital wireless communication technology and software technology. Therefore, the main topic of SDR is how a hand-held device can dynamically change

0-7803-7661-7/03/\$17.00@2003 IEEE the software control module to match the wireless communication system, in other words, smoothly hand-over to a different system.

The Joint Tactical Radio System (JTRS) [5] has proposed a Software Communication Architecture (SCA) specification [6] that defines clearly the architecture of software technology in SDR. Referring to the SCA specification, "SDR Forum Technical Report 2_1" [4] and related documents [7][8], we will propose a software prototyping which is called SDRsim in this paper. It provides high-level functional models that are capable of being mapped into specific software-defined devices such as hand-held, mobile, and base station applications. SDRsim is a prototyping platform which provides modularity, and requires minimal implementation effort to accommodate the SDR device. Due to the complexity and associated cost of building modern computer systems, simulation is often the only practical way to test architectural ideas and access system performance.

This paper is organized as follows: Section 2 proposes the SDRsim software architecture. Section 3 discusses the design and analysis of SDRsim. Section 4 proposes the implementation mechanisms of SDRsim. Section 5 surveys some related works. Finally, Section 6 makes some conclusive remarks.

\section{The overview of the SDRsim software architecture}

Some important technologies and software components are needed to support SDR technology according to the requirements of SDR concept. Figure 1 shows the layered elements in the SDRsim software architecture. Its functionalities will be described respectively as follows:

\footnotetext{
We thank Industrial Technology Research Institute grant T1-90028, the National Science Council grant 90-2213-E-009-160, 91-2520-S-009-007 and the Ministry of Education Program of Excellence Research 89-EFA04-1-4.
} 


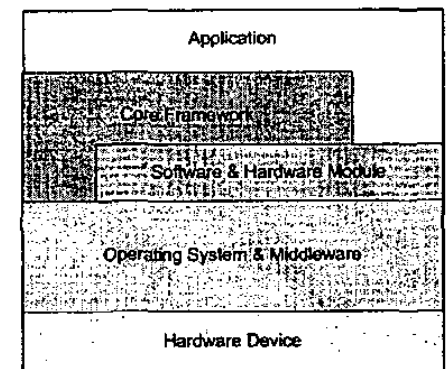

Figure1. The SDRsim software architecture

\subsection{Application}

Application may be SDR utilities or be the end-user applications which are developed by the third-party commercial parts. Application could be e-mail utilities, Java objects (MIDlet, PersonalJava), user interfaces etc. Application can directly use Core Framework's functionalities or invoke Software \& Hardiware Module to accomplish jobs.

\subsection{Core Framework}

Users can invoke the provided objects (such as Domain Manager, Resource Manager, and File Manager etc.)in Core Framework to control SDRsim. For example, Core Framework can be used to switch among various protocols so that SDRsim can roam on multiple modes of personal communication systems. Core Framework: consists of:

- Base CORBA [9] interfaces (Configure, Life, Test and Port)are inherited by Software \& Hardware Module to provide the functionalities for controlling and communicating with Software \& Hardware Module.

- Functional elements (like Domain Manager, Resource Manager and File Manager etc.) provide framework control of all system operations via CORBA interfaces.

- Domain Profile describes the properties of Software \& Hardware Module and the information of mode configuration, resource description etc.

\subsection{Software \& Hardware Module}

Software Module provides the standard interfaces for the control and communication behavior which includes repeater, link, bridge, network, router, and gateway operations. Software Module relies on commercial components to support multiple unique serial and network interfaces. Reliable transport mechanisms may include error checking and correction at the network and serial interface level. Possible serial and network physical interfaces include RS232, RS422, RS423, RS485, Ethernet, 802.x, and others. In order to support the standard interfaces, various low-level network protocols may be used. They may include PPP, SLIP, CSLIP, LAPX, and others. Using these protocols, other protocols such as IP, TCP/UDP, and X.25 can be added to provide network connection. Software Module also includes typical security operations which provide key fill, . key management, programmable security device control, and software integrity and authentication.

Hardware Module is also a software component. It provides the functionalities such as modulation, CODEC, interleaving, and equalization etc. It also can be downloaded from content providers and dynamically loaded and executed. Hardware Module includes two major elements: the driver and signal processing algorithm software. They are described as follows:

- Driver: With respect to different communication protocols, they need differential drivers which provide the functionalities of controlling Hardware Device. Therefore, drivers download is needed when starting the operation of changing or establishing communication protocols.

- Signal processing algorithm software: Signal processing algorithm is used in the reconfigurable device such as DSP (Digital Single Processing). Like drivers, the single processing algorithm software may need to be downloaded when executing the operation of changing or establishing communication protocols. Therefore, the single processing algorithms have to allow dynamically downloading and loading into the reconfigurable device. Binding up the needed single processing algorithms into a software object is needed for effective downloading.

\subsection{Operating System \&.Middleware}

In order to provide the powerful execution environment for SDRsim, it is needed to support the real-time Operating System and Middleware which can supply the following functionalities:

- Real-time requirement: SDRsim provides the functionalities of configure mode, establish mode, change mode etc. It is able to accomplish these jobs in a constrained time. Therefore, a real-time Operating System is required.

- Minimal memory requirement: For general hand-held device, the storage size is limited because of the concern for the 
volume and cost. In order to utilize the constrained memory size, the size of Operating System and Middleware which used in SDRsim will suit to the storage size.

- Services providing: According to "SDR Forurn Technical Report 2_l" [4], the standard interfaces and API are already proposed. The implemented functionalities of these interfaces and APIs all execute on the CORBA environment. Therefore, a suited CORBA environment will be provided.

The real-time Operating System used in SDRsim has to provide the functionalities to deal with the time-constrained problems. These functionalities are depicted as follows:

- Thread/Process handling: Operating System controls the scheduling operation which utilizes the property level of thread and process. The scheduling operation is an important feature which can facilitate accomplishing jobs before the deadline.

- Resource management: Operating System can supply the resource status management and provide the functionalities for querying and setting system resources (likes memory and disk). It helps to allocate the applications with needed resources which are used to complete the time-constrained jobs.

- High-performance I/O system: The high-performance $\mathrm{V} / \mathrm{O}$ rate is to ensure the constrained-timed data transmission be completed before the deadline.

- Providing high-performance network for data transmitting.

- Supported processor.

- Supported developing language.

Based on a real-time Operating System, the real-time CORBA environment can be used with a high quality. The real-time CORBA environment in SDRsim has to meet following criteria:

- Based on a real-time Operating System.

- Providing a real-time ORB(Object Request Broker).

- Providing high-performance I/O.

- Providing scheduling operation which can be integrated in ORB.

- Providing high-performance network for data transmitting.

- Supported developing language.

The following contents put the emphasis on the survey of the COTS (Commercial -Of-The-Shelf) real-time Operating System and CORBA environment. It is needed to combine a real-time Operating System and CORBA environment together to supply SDRsim. Table 1 includes the information about the COTS real-time Operating System.

According to "Measuring OS Support for Real-time CORBA ORBs" [10], the traditional Operating Systems such as Windows NT, Solaris, and Linux are not competent to meet the real-time requirements. The reasons are that the non-real-time Operating Systems cannot promise to accomplish the task in a constrained time. Therefore, we have to find the specified Operating System which can provide the real-time functionalities. The following depicts several COTS real-time Operating Systems and compare these Operating Systems with the conditions addressed above.

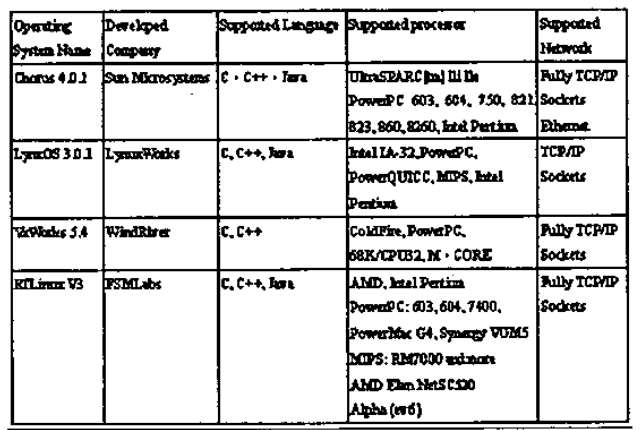

Tablel. The COTS Real-Time Operating System

Three CORBA technologies that can be combined with a real-time Operating System together to supply SDRsim are depicted as follows:

- TAO [11]: It is the first developed real-Time CORBA environment. It can be portable on Windows-related operating system which contains UNIX, LynxOS, VxWorks, QnX Neutrino, and Chorus ClassiX 4.0 etc.

- VisiBroker [12]: It is developed by Inprise/Borland company. It can be portable on several real-time Operating Systems such as LynxOS and VxWorks etc.

- Orbix [13]: It is developed by IONA Company. Although it does not provide any real-time CORBA environments, it has already proposed several subjects to develop the real-time CORBA environment which can be combined with real-time Operating Systems.

Besides these real-time CORBA environments, OMG [14] formulates the Embedded CORBA specification which is exactly a Minimal-CORBA environment which tries the best to simplify the functionalities of the CORBA environment to satisfy the constrained storage of SDR device. 


\subsection{Hardware Device}

The elements belonging to Hardware Device may be the general hardware devices (such as Ethernet Card, Audio Card etc.) or the reconfigurable hardware devices (such as DSP device). Hardware Device is the physical implementation of SDRsim which will have values for the relevant attributes based on the system's physical requirements and the procurement performance requirements.

\section{Design and analysis}

This section focuses on the SDRsim design flow which is a representation of SDRsim that rationalizes, arranges, and connects components to produce the desired functionality. With respect to the design and analysis of SDRsim, dividing the design and analysis flows into four parts can help to describe more clearly. The first part is to identify the functional elements included in the SDRsim software architecture. In the second and third parts, use UML (Unified Modeling Language) technology to design the Use Case and Sequence Diagrams which are central to model the behavior of SDRsim

3.1 The functional elements of the SDRsim software architecture

Figure 2 is proposed to present the design of several functional elements and the relationships among these functional elements of the SDRsim software architecture. The SDRsim software architecture includes the functional elements which can be separated into five individual layered components: Application, Core Framework, Software \& Hardware Module, Operating System \& Middleware and Hardware Device. Application, Operating System and Middleware are all the COTS software that satisfies the requirements of the SDR concepts which are described above. Therefore, the design flows will focus on the functional elements of Core Framework and Software \& Hardware Module.

The functional elements belonged to Core Framework depicted as follows:

- Domain Manager: Domain Manager is the central administrated element in Core Framework. It is responsible for controlling the functionalities of Core Framework and assigning jobs to the corresponding elements. It is also responsible for monitoring the execution status of Core Framework.

- Resource Manager: Resource Manager is responsible for managing the resource status of Hardware Device and providing the functionalities for querying and setting system resources (like memory and disk). It is also responsible for detecting the error status of Hardware Device and reporting this status back to Domain Manager. Resource Manager provides a set of functionalities for Software Module Manager, Hardware Module Manager and Application to allocate the system resources.

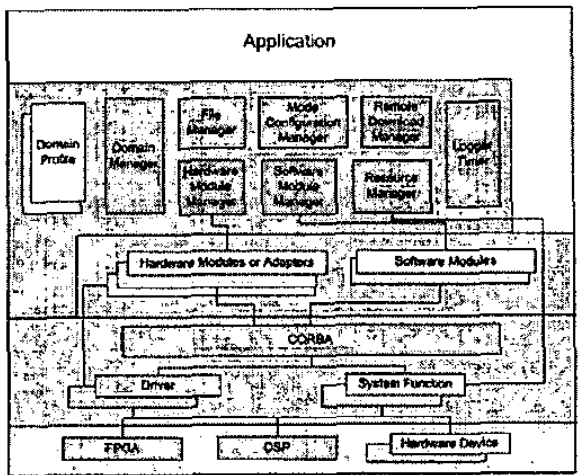

Figure2. Functional elements in the SDRsim software architecture

- File Manager: File Manager is responsible for managing the files and file systems in SDRsim.

- Remote Download Manager: Remote Download Manager is responsible for downloading the software which includes Software Module, Hardware Module and Application.

- Mode Configuration Manager: Mode Configuration Manager provides the status lists of Hardware Module and Software Module. It is responsible for detecting the status of currently used communication environment, and then deciding if it has to execute the operation of changing mode or not. It also provides the lists of available communication environments which can be used right now. When receiving the command of changing mode, Mode Configuration Manager will estimate the status of currently used communication environment to check if it can accomplish this operation in the constrained time.

- Software Module Manager: Software Module Manager is responsible for managing and monitoring the status of Software Module. It is also responsible for reconfiguring Software Module.

- Hardware Module Manager: Hardware Module Manager is responsible for managing and monitoring the status of Hardware Module. It is also responsible for reconfiguring Hardware Module. 
- Logger: A Logger is utilized by SDRsim to store informational messages. These informational messages are also called as $\log$ records in this paper. The Logger is responsible for recording the execution messages and exception messages. It is implemented according to CORBA Log Service.

- Timer: A Timer provides operations for synchronizing time within radio as well as for creating and managing time-based events. The Timer is based on CORBA Time Service.

- Domain Profile: Software Module and Hardware Module make up an SDRsim domain which is described by a set of files that are collectively called a Domain Profile. All of the descriptive data in the Domain Profile is expressed in the XML (eXtensible Makeup Language) vocabulary which describes a distinct aspect of Software Module and Hardware Module. These files describe identifies, capabilities, properties, inter-dependencies, and locations of Software Module and Hardware Module. They also describe the records of services which subscribed from content providers.

To facilitate controlling Software Module and Hardware Module, the standard interfaces are needed to be provided. Both Software Module and Hardware Module provide the standard interfaces which are formatted as IDL (Interface Definition Language) type. They all execute on the CORBA environment. The standard interfaces are depicted as follows:

Configure: provide the configuring operations with the specified properties. Life: provide controlling functionalities such as startup and stop operations. Test: provide the self-test functionalities with the specified properties. Port: provide the co-operation functionalities which can be put into work together with other Software Module and Hardware Module.

\subsection{UML design for SDRsim}

With respect to design the operations of SDRsim, using UML technology is suited to describe the design flow more clearly. The UML is a graphical language for visualizing, specifying, constructing, and documenting the artifacts of software-intensive system. The UML gives a standard way to write the system's blueprints, covering conceptual things, such as system's functions, as well as concrete things, such as system's classes written in a specific programming language, and reusable software components. Core Framework is the most important component which provides the functionalities for controlling all the components in SDRsim, and monitors their execution status. Therefore, the design flow will base on Core Framework and then expand to SDRsim.

Section 3.2.1 uses UML to design the Core Framework Use Case Diagrams which show a set of use cases, actors and their relationships. It is applied to illustrate the static use case view of a system. Section 3.2.2 devises the Core Framework Sequence Diagrams which show a set of functional elements of SDRsim and the messages sent and received by those functional elements.

\subsubsection{The Core Framework Use Case Diagrams}

Twelve use cases and five actors are included in this diagram. The five actors are described as follows:

- OA\&M: It may be users of hand-held devices or system venders.

- Content Provider: provide applications and services to be downloaded and supply the functionalities for executing services and applications.

- Software Module, Hardware Module and Hardware Device

Figure 3 is the Use Case Diagrams of Core Framework. It shows the twelve use cases. These use cases will be described as follows:

- Bootup: boot-up or reconfigure SDRsim.

- Remote Download: connect with content providers and download the specified software which includes Software Module, Hardware Module and Application.

- Manage Resource: manage the resource status of Hardware Device, provide the functionalities for querying and allocating system resources (like memory and disk).

- Configure Software Module: configure Software Module's status and functionalities.

- Configure Hardware Module: configure Hardware Module's status and functionalities.

- Mode Configuration Management: provide the status lists of the current used Software Module, Hardware Module and communication environment. It is also responsible for executing the operation of changing mode.

- Establish Mode: establish the specified communication environment.

- Release Mode: release the current used communication environment.

- Change Mode: change the current used communication environment to the specified one.

- Environment Detection: detect the 
available communication environment which can be used right now.

- Status Report: provide the functionalities for receiving the messages which come from Software Module and Hardware Module.

- Shutdown: OA\&M shutdown or release SDRsim.

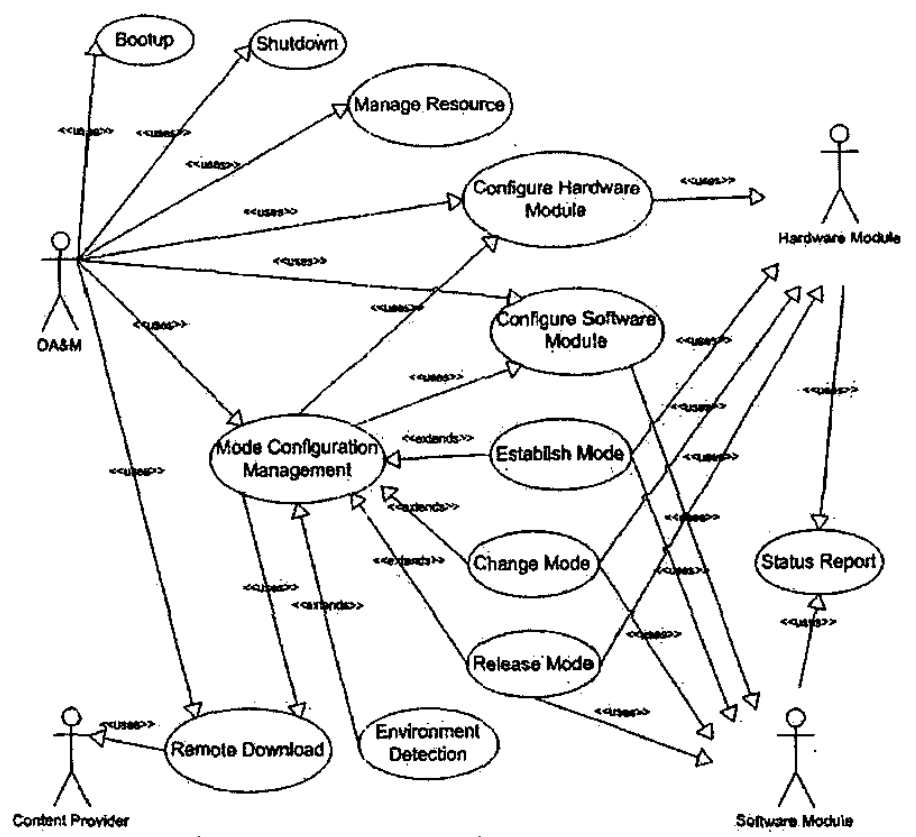

Figure3. Core Framework Use Case Diagrams
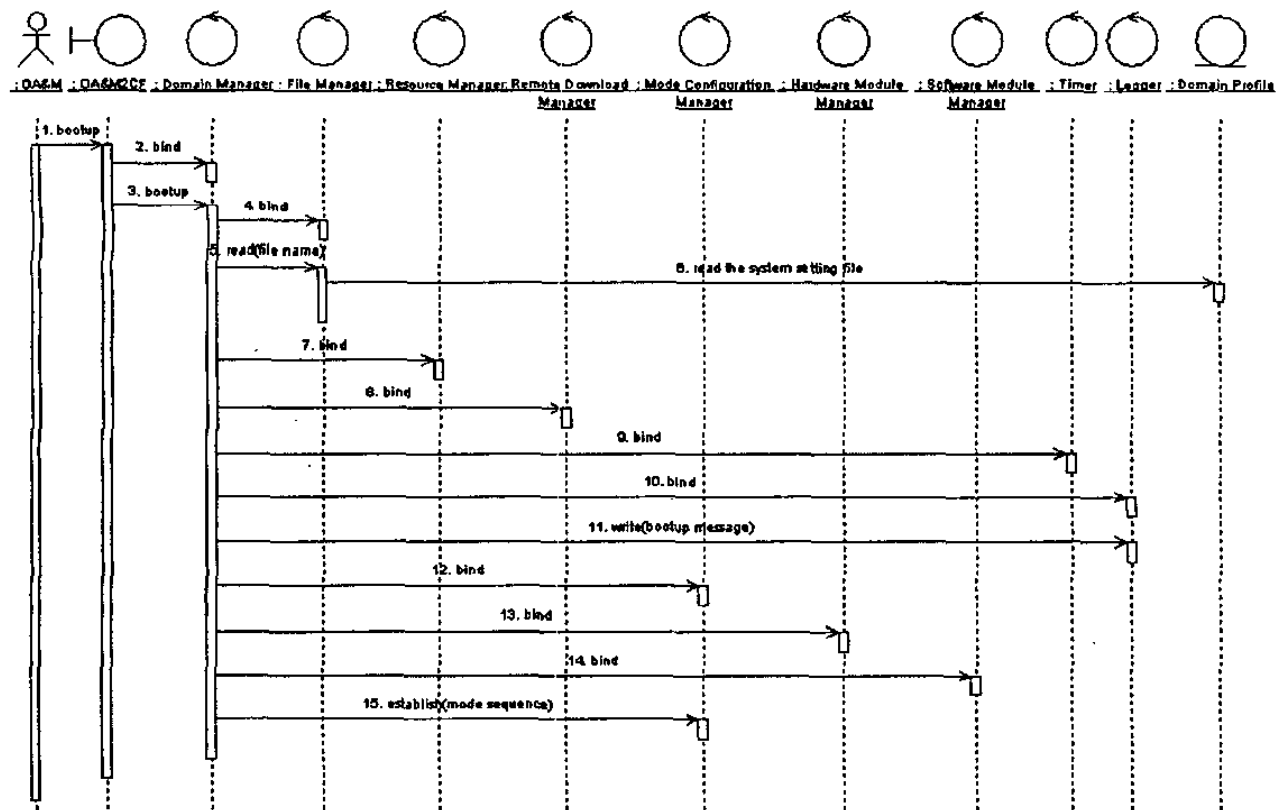

Figure4. Bootup Sequence Diagram

3.2.2 The Core Framework Sequence Diagram

Sequence Diagrams show a set of functional elements and the messages sent and received by those functional elements which are typically named or anonymous instances of classes or collaborations, components, and nodes. The Core Framework Sequence Diagrams 
present the specified use case's behavior in the Core Framework Use Case Diagrams. They are described in the following process in which the execution steps are depicted. The example of the Bootup Sequence Diagram represents the behavior of "Bootup" use case in the Core Framework Use Case Diagrams. The example is presented in Figure 4 and the execution steps are depicted as follows:

1. OA\&M may be actions caused by users of hand-held devices or system venders. It starts the bootup operation of Core Framework.

2. OA\&M binds Domain Manager.

3. OA\&M demands that Domain Manager execute the bootup operation.

4. Domain Manager binds File Manager .

5. Domain Manager demands that File Manager read the system setting file which includes the previous system configuration information.

6. File Manager reads the system setting file.

7. Domain Manager binds Resource Manager.

8. Domain Manager binds Remote Download Manager.

9. Domain Manager binds Timer.

10. Domain Manager binds Logger

11. Domain Manager demands that Logger write the $\log$ records of bootup operation.

12. Domain Manager binds Mode Configuration Manager.

13. Domain Manager binds Hardware Module Manager.

14. Domain Manager binds Software Module Manager.

15. Domain Manager demands that Mode Configuration Manager establish the communication environment according to the mode sequence properties.

\section{Implementation}

SDRsim is implemented according to the design flow in Section 3. The features and functionalities of SDRsim have been implemented as primitives in a "class library" that focuses on the design flow which bases on Core Framework, Software Module and Hardware Module.

The implemented "class library" of Core Framework are included in sdr.cf package. All implemented classes use the Java programming language [15] and provide standard interfaces which are formatted as IDL type. All implemented classes execute on the VisiBroker CORBA middleware and RTLinux [16] platform.

For example, the implemented classes and interfaces in the sdr.cf.domainManager package provide the functionalities of Domain
Manager.

The Domain Profile is consisted of five kinds of files which are Software Module Descriptor, Hardware Module Descriptor, Mode Assembly Descriptor, Resource Descriptor, and Available Mode Descriptor file.

A Software Module Descriptor file contains information about the interfaces that the specific Software Module provides and/or uses. A Hardware Module Descriptor file contains information about the interfaces that the specific Hardware Module provides and/or uses. A Mode Assembly Descriptor file contains information about the specific Software Module and Hardware Module that make up a communication protocol. A Resource Descriptor file contains information about allocating and de-allocating system resources to a processor, DSP, FPGA, and other resources which will report their presence in the system through the Resource Manager. An Available Mode Descriptor file contains information about the available communication environments that can used right away, Mode Configuration Manager will maintain this file and use it when establishing or changing mode. All of the descriptive data in Domain Profile is expressed in the XML vocabulary.

The implementing elements of Software Module and Hardware Module focus on the standard interfaces which are formatted as IDL type. All elements can be implemented in several programming languages which are supported by the CORBA environment.

Figure 5 depicts the functional elements of Core Framework. Figure 6 depicts the system operations of Software Module and Hardware Module designing. The system developer can use SDRsim to automatically construct the basic functions of Software Module and Hardware Module according to the standard interfaces which are described above.

\section{Related works}

The past and present projects have sponsored the research in SDR-related technologies include IBMS (Integrated Broadband Mobile System) [17], MOBIVAS (downloadable MOBIle Value Added Service) [18], TRUST (Transparently Reconfigurable UbiquitouS Terminal) [19] and so on.

The objective of IBMS project is the integration of low and high data rate services for outdoor and inhouse mobile communication system based on ATM transmission.

The MOBIVAS project is proposed by IST-1999-10206 and the overall objective of MOBIVAS is to develop architectural approaches and prototypical implementations of 
integrated software platforms and systems, which will enable the flexible provision of Value-Added Services (VAS) in mobile communication networks. These platforms should be adaptable to different network services and technologies and will constitute new opportunities for third-party Value-AddedService Providers (VASPs).

The IST project TRUST that attempts to rationalize the "seamless wireless utopia" by studying the real user requirements for reconfiguring terminals and then creating realistic working scenarios.

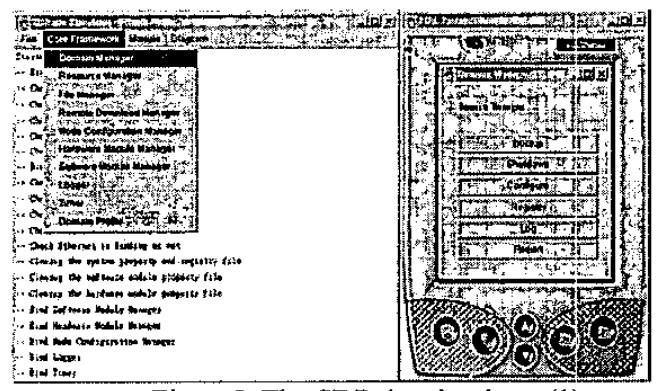

Figure5. The SDRsim simulator (1)

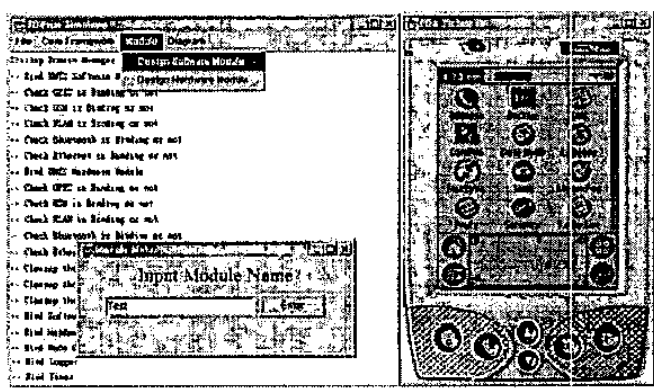

Figure6. The SDRsim simulator (2)

\section{Conclusions}

This paper represents a pc-based tool for simulation and rapid control prototyping of SDR system, based on industry standard architecture and COTS technologies. We propose a software prototyping which is called SDRsim. It provides high-level functional models that are capable of being mapped into specific software-defined devices such as hand-held, mobile, and base station applications. SDRsim is a prototyping platform which provides modularity, and requires minimal implementation effort to accommodate the SDR device.

We implement the SDRsim simulator which is developed by the COTS software technologies such as RTLinux, VisiBroker CORBA environment and Java technology etc. The features and functions of this SDRsim simulator have been implemented as primitives in a class library that focuses on the design flows which are based on the requirements of SDR concept.

\section{References}

[1]. J.Mitola, "Software Radio: Survey, Critical Evaluation and Future Directions", IEEE National Telesystems Conference, pp. 13-15-13-23, May 1992.

[2]. J.Mitola, "The Software Radio Architecture", IEEE Communication Magazine, Vol. 33, No. 5, pp. 26-37, May 1995.

[3]. The SDR Forum (formally MMITS), http://www.sdrforum.com

[4]. SDR Forum Technical Report 2_1.

http://www.sdrforum.com

[5]. Joint Tactical Radio System (JTRS), http://www.jtrs.saalt.army.mil

[6]. Modular Software-programmable Radio Consortium, "Software Communications Architecture Specification", MSRC-5000SCA.

[7]. Gi-Ping Lee, Yue-Shan Chang, Shyan-Ming Yuan, "A Software Framework for Software Radio," in Proc. of WCC2000/ICCT2000, Beijing, China, August 21-25, 2000. pp. 1102-1105.

[8]. Yuan-Deng Chuang, Yue-Shan Chang, Shyan-Ming Yuan, "A Framework for Integrating MExE - with SDR's Software Architecture," Networks 2002(Joint conference ICWLHN2002 and ICN2002), Aug. 26 - 29, 2002, Atlanta, Georgia, USA, pp. - .

[9]. The Object Management Group , The Complete CORBA/IIOP 2.3.1 Specification, OMG document formal/99-10-07;

[10]. David L. Levine, Sergio Flores-Gaitan, Christopher D. Gill and Douglas C. Schmidt, "Measuring OS Support for Real-time CORBA ORBs", Proceedings of the Fourth IEEE International Workshop on Object-oriented Real-time Dependable Systems (WORDS'99), Santa Barbara, California, January 27-29, 1999

[11]. TAO, "http://www.cs.wustl.edu/ schmidt/ TAO-intro.html"

[12]. VisiBroker, Borland Corp. http://www.borland.com/bes/ visibroker/

[13]. Orbix, http://www.orbix.gq.nu/

[14]. The Object Management Group, http://www.omg.org"。

[15]. Sun Microsystems, http://java.sun.com

[16]. FSMLabs, RTLinux (Real Time Linux), http://ssmlabs.com/community/

[17]. BMS(Integrated Broadband Mobile System) project, http://www-ibms.ee.tu-berlin.de/

[18]. IST-1999-10206 MOBIVAS (downloadable MOBIle Value Added Service) Deliverable D-2.1.2 Requirements for general architecture \& interface specification.

[19]. IST-2000 TRUST (Transparently Reconfigurable Ubiquitous Terminal), http://www.ist-trust.org/title.html 